\title{
Electronic states of epitaxial thin films of $\mathrm{La}_{0.9} \mathrm{Sn}_{0.1} \mathrm{MnO}_{3}$ and $\mathrm{La}_{0.9} \mathrm{Ca}_{0.1} \mathrm{MnO}_{3}$
}

\author{
J. Gao, S. Y. Dai, and T. K. Li \\ Department of Physics, The University of Hong Kong, Pokfulam Road, Hong Kong
}

(Received 23 December 2002; published 15 April 2003)

\begin{abstract}
Structure, transport properties, and electronic structure of epitaxial thin films $\mathrm{La}_{0.9} \mathrm{Sn}_{0.1} \mathrm{MnO}_{3}$ and $\mathrm{La}_{0.9} \mathrm{Ca}_{0.1} \mathrm{MnO}_{3}$ have been experimentally studied. According to the Hall-effect measurement, $\mathrm{La}_{0.9} \mathrm{Sn}_{0.1} \mathrm{MnO}_{3}$ is an $n$-type conductor in the metallic state due to the substitution of Sn for La. X-ray photoelectron spectroscopy spectra revealed a shift of the Fermi level and the Mn ionic core level of $\mathrm{La}_{0.9} \mathrm{Sn}_{0.1} \mathrm{MnO}_{3}$ in comparison with $\mathrm{La}_{0.9} \mathrm{Ca}_{0.1} \mathrm{MnO}_{3}$. The difference between the Mn-2 $p$ spectra of $\mathrm{La}_{0.9} \mathrm{Sn}_{0.1} \mathrm{MnO}_{3}$ and $\mathrm{La}_{0.9} \mathrm{Ca}_{0.1} \mathrm{MnO}_{3}$ implies that $\mathrm{Mn}$ ions in the former are at a $\mathrm{Mn}^{2+} / \mathrm{Mn}^{3+}$ mixed-valence state, which is significantly different from the divalent-element-doped manganese oxides, where the $\mathrm{Mn}$ ions are in the mixed-valence state of $\mathrm{Mn}^{3+} / \mathrm{Mn}^{4+}$.
\end{abstract}

DOI: 10.1103/PhysRevB.67.153403

PACS number(s): 73.21. $-\mathrm{b}, 73.50 .-\mathrm{h}, 73.90 .+\mathrm{f}, 75.70 .-\mathrm{i}$

Numerous studies have focused on the family of doped manganese perovskite oxides $R_{1-x} A_{x} \mathrm{MnO}_{3}$ recently, where $R$ is a trivalent rare-earth element and $A$ is a divalent element such as $\mathrm{Ca}, \mathrm{Sr}, \mathrm{Ba}$, and $\mathrm{Pb}$, etc. In this kind of material, an insulator-to-metal transition occurs that is usually accompanied by a paramagnetic-to-ferromagnetic transition, and a colossal magnetoresistance (CMR) effect takes place near the Curie temperature. ${ }^{1}$ It is believed that these phenomena are due to the double exchange interaction and Jahn-Teller effect. $^{2}$ These oxides are of great importance both for scientific study and practical applications. Unfortunately, the low working temperature and considerable noise has restricted most commercial applications. It has been experimentally proven that the conventional divalent-ion-doped manganite exhibits a $p$-type conductivity. However, we can change the character of the charge carrier in the compound by replacing $\mathrm{La}^{3+}$ with $\mathrm{Sn}^{4+}$. A combination of the $n$ - and $p$-type oxides could form a $p$ - $n$ junction, producing a functional device for all oxides. It could be used in a wide range of temperature, and operated with low noise and high sensitivity. This is why the recently developed $n$-type La-Sn-Mn-O compound has attracted much research interest. ${ }^{3-5}$

In this paper we report the electronic structure and transport properties of $\mathrm{La}_{0.9} \mathrm{Sn}_{0.1} \mathrm{MnO}_{3}$ (LSMO). Hall-effect measurements have suggested $n$-type conductivity in LSMO. Compared to $p$-type $\mathrm{La}_{0.9} \mathrm{Ca}_{0.1} \mathrm{MnO}_{3}$ (LCMO) thin films, a chemical shift of the Fermi level and a widening of the valence band were observed in LSMO based on the study of $x$-ray photoelectron (XPS) spectra. From an analysis of the Mn- $2 p$ spectra, Mn ions in the LSMO film could be at a $\mathrm{Mn}^{2+} / \mathrm{Mn}^{3+}$ mixed-valence state, instead of $\mathrm{Mn}^{3+} / \mathrm{Mn}^{4+}$ as in the LCMO film.

The thin films were fabricated by using pulse laser deposition. The targets of LSMO and LCMO were prepared by the conventional solid-state reaction method. The stoichiometric mixture of high-purity $\mathrm{La}_{2} \mathrm{O}_{3}, \mathrm{Mn}_{3} \mathrm{O}_{4}$, and $\mathrm{SnO}_{2}$ or $\mathrm{CaCO}_{3}$ powder was milled, pressed, and presintered three times. Then the powder was pressed into a disk of $50 \mathrm{~mm}$ in diameter and sintered at $1203 \mathrm{~K}$, for LSMO, and $1573 \mathrm{~K}$, for LCMO. Single-crystal $\mathrm{SrTiO}_{3}$ (100) was used as a substrate. The deposition temperature was about $900 \mathrm{~K}$, measured by a $k$-type thermocouple inserted into the heater block. The deposition was performed in pure oxygen at a pressure of $2 \times 10^{-2}$ mbar. The power of the pulse laser beam was about $300 \mathrm{~mJ}$ and the frequency was $10 \mathrm{~Hz}$. The growing process was halted when the thickness of the film was about $300 \mathrm{~nm}$ and then the film was annealed at about $1073 \mathrm{~K}$ for about 4 $\mathrm{h}$ in air.

All the grown films were examined by a SIEMENS D5000 x-ray diffractometer with $\mathrm{Cu}-K \alpha$ radiation. Figure 1 presents a typical $x$-ray-diffraction spectrum of the LSMO film. No peaks from the secondary phases such as tin oxides are visible, clearly demonstrating that the film is of single phase and exhibits a perfect (001) orientation. The diffraction peaks agree well with that of the $A B \mathrm{O}_{3}$ structure. The outof-plane lattice constant of LSMO was measured to be $3.8748 \AA$. The partial substitution of $\mathrm{Sn}$ for $\mathrm{La}$ in $\mathrm{LaMnO}_{3}$ induces minor lattice constrictions due to the different ionic radii of $\mathrm{Sn}$ and $\mathrm{La}$. The lanthanum or tin might be slightly deficient due to a loss during the high-temperature process. However, the deficit of La or Sn was found very small, as reflected by energy-dispersive $\mathrm{x}$-ray analysis. The average composition for LSMO films is La:Sn:Mn=0.88:0.086:1, approaching stoichiometry.

The temperature dependence of the resistivity was measured by a standard four-probe method in the range of 4.2$300 \mathrm{~K}$ under external magnetic fields of $0-4 \mathrm{~T}$. The rather

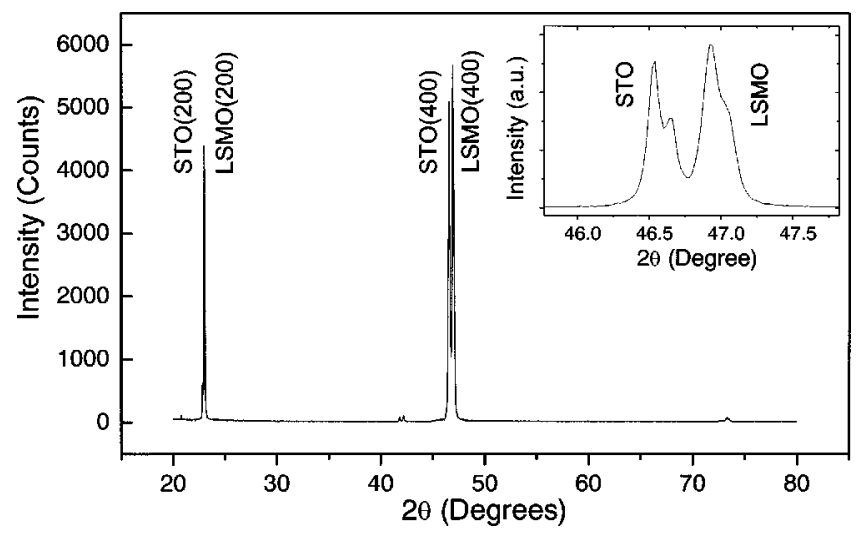

FIG. 1. Typical x-ray-diffraction pattern of the $\mathrm{La}_{0.9} \mathrm{Sn}_{0.1} \mathrm{MnO}_{3}$ epitaxial thin films. The insets show the enlarged (400) peaks. 


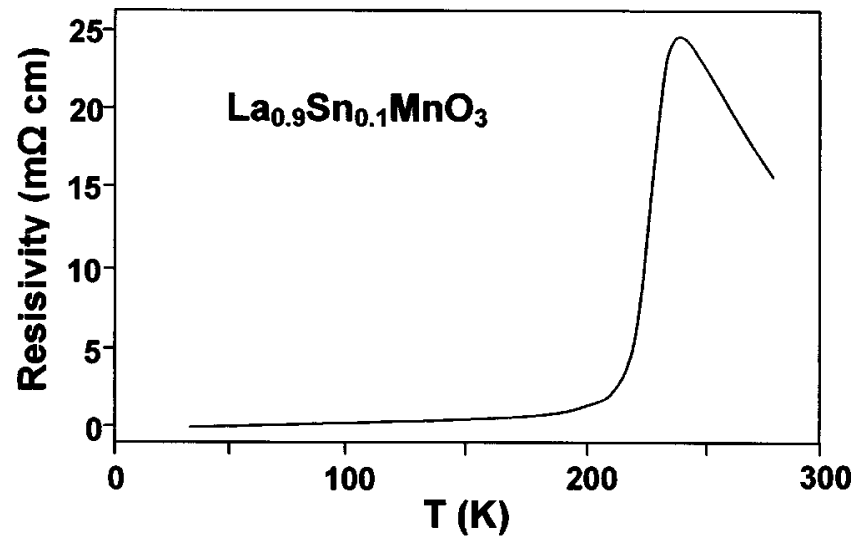

FIG. 2. The temperature dependence of resistivity of one of our LSMO films.

sharp transition implies the high purity in the phase structure, as seen in Fig. 2. The experimental results revealed that these films exhibited a standard CMR behavior and the magnetic resistance ratio, $[\rho(H)-\rho(0)] / \rho(0)$, was about $85 \%$ near the Curie temperature $(\sim 243 \mathrm{~K})$. Its $\rho$ - $T$ dependence is similar to that of LSMO thin films and bulk material. ${ }^{5}$ The resistivity of these LSMO films is about $10^{-2}-10^{-3} \Omega \mathrm{cm}$, remarkably lower than that of La-deficient $\mathrm{La}_{x} \mathrm{MnO}_{3}$ ( $\rho$ $=8.6 \Omega \mathrm{cm}){ }^{6}$ As reported by Gupta et al. and McGuire et al., the Curie temperature of the $\mathrm{La}_{0.9} \mathrm{MnO}_{3}$ thin films was $\sim 130 \mathrm{~K}^{6,7}$ The maximum value of $T_{c}$ for thin films of $\mathrm{La}_{0.9} \mathrm{MnO}_{3-\delta}$ with an annealing in oxygen at $850{ }^{\circ} \mathrm{C}$ could increase to $\sim 180 \mathrm{~K}$, which is still much lower than that of our samples ( $T_{c} \sim 243 \mathrm{~K}$ for our LSMO films). Such a comparison suggests that our films were not formed as a mixture of $\mathrm{La}_{0.9} \mathrm{MnO}_{3}$ and $\mathrm{SnO}_{2}$. The $\mathrm{Sn}$ atoms in our LSMO films should occupy the La site.

It is well known that the partial substitution of $\mathrm{La}^{3+}$ by a divalent element in $\mathrm{LaMnO}_{3}$ may introduce a mixture system of $\mathrm{Mn}^{3+}$ and $\mathrm{Mn}^{4+}$ ions. The double exchange interaction between Mn ions, combined with the Jahn-Teller distortion model, qualitatively explained the CMR behavior and related transitions. ${ }^{2}$ That is to say that the $\mathrm{Mn}^{3+}$ ion has an electron configuration $t_{2 g}^{3} e_{g}^{1}$. The low-energy $t_{2 g}^{3}$ triplet state contributes a local spin of $S=\frac{3}{2}$, and the $e_{g}^{1}$ state electron is either itinerant or localized, depending on the local spin orientation. The $e_{g}^{1}$ electron hopping between $\mathrm{Mn}^{3+}$ and $\mathrm{Mn}^{4+}$ and its dependence on magnetic field also qualitatively describe the mangnetoresistance behavior. In order to explain the insulatorlike behavior at a higher temperature, the Jahn-Tellertype polaron effect has to be considered in the double exchange model. If a part of the $\mathrm{La}^{3+}$ ions was substituted by four or five valence elements such as $\mathrm{Sn}, \mathrm{Te}, \mathrm{Sb}$, etc., a mixed-valence system of $\mathrm{Mn}^{3+}$ and $\mathrm{Mn}^{2+}$ ions could be observed and the electron-doping feature would be expected. For LSMO films, XPS revealed that the binding energy for Sn- $3 d_{5 / 2},-3 d_{3 / 2}$, and $-4 d$ were $486.44,494.88$, and 25.81 eV respectively, which are very close to that of $\mathrm{SnO}_{2} \cdot{ }^{7}$ Thus the experiments confirmed that the $\mathrm{Sn}$ ions in the $\mathrm{La}_{0.9} \mathrm{Sn}_{0.1} \mathrm{MnO}_{3}$ sample are in the four-valence state.

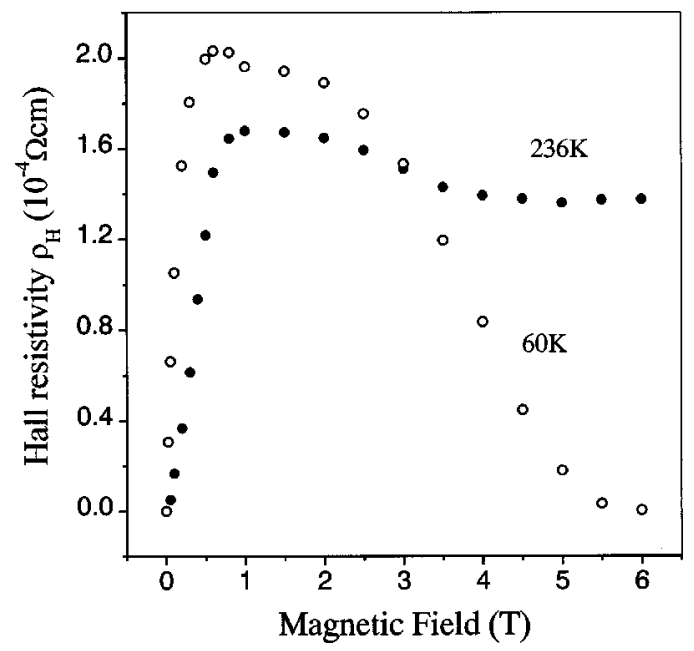

FIG. 3. Hall resistivity vs magnetic-field curves for $\mathrm{La}_{0.9} \mathrm{Sn}_{0.1} \mathrm{MnO}_{3}$ at temperatures 60 and $236 \mathrm{~K}$.

In order to understand the picture of charge transport in the sample, measurements on the anomalous Hall effect were performed on $\mathrm{La}_{0.9} \mathrm{Sn}_{0.1} \mathrm{MnO}_{3}$ films using an Oxford superconducting magnet system and a dc current source. In order to gain correct Hall voltage and avoid the magnetic hysteresis effect in low fields, the Hall voltage was obtained by proper averaging of the signals measured at reversal directions of the measuring current and field. In practice, we took six sets of Hall voltage in the sweeping field (from 6 to -6 $\mathrm{T}$ ) and the Hall voltage was obtained by averaging the last five sets of data. Then we calculated the Hall resistivity based on the dimension of sample and measuring dc current $(5 \mathrm{~mA})$. Figure 3 shows the function curves of the measured Hall resistivity vs external magnetic field at 60 and $236 \mathrm{~K}$, respectively.

The anomalous Hall effect was first discovered in ferromagnetic metal, and then observed in manganese perovskite oxides. ${ }^{8}$ In general, for a ferromagnetic material in an applied magnetic field $H$, the dependence of transverse resistivity vs field can be written as

$$
\begin{gathered}
\rho_{x y}=R_{0} B+R_{A} \mu_{0} M, \\
B=\mu_{0}[H+(1-N) M],
\end{gathered}
$$

where $R_{0}$ and $R_{A}$ are ordinary and anomalous Hall coefficients, respectively. $M$ is the magnetization and $N$ is the demagnetization factor. The Hall resistivity is about $10^{-4} \Omega \mathrm{cm}$, that is, close to the Hall resistivity value of $\mathrm{Tl}_{2} \mathrm{Mn}_{2} \mathrm{O}_{7}$ and $\mathrm{La}_{2 / 3} A_{1 / 3} \mathrm{MnO}_{3}(A=\mathrm{Ca}, \mathrm{Sr})$ thin films. ${ }^{9}$ At a low field, the $\rho_{H}$ increases steeply with the field, reaches a maximum at about 0.6 and $1 \mathrm{~T}$ for temperatures 60 and 236 $\mathrm{K}$, respectively, and then decreases with an approximate linear slope. The initial part of $\rho$ vs $H$ curves is dominated by the magnetization of the sample rather than by the external magnetic field. At high fields, the magnetization is saturated and is field independent. The slope $d R_{H} / d B$ is given by ordinary Hall contribution induced by Lorentz forces on charge carriers, $R_{0}=t d R_{H} / I d B$, and the effective carrier density $n_{\mathrm{eff}}=1 / e R_{0}$. The experimental results on LSMO 


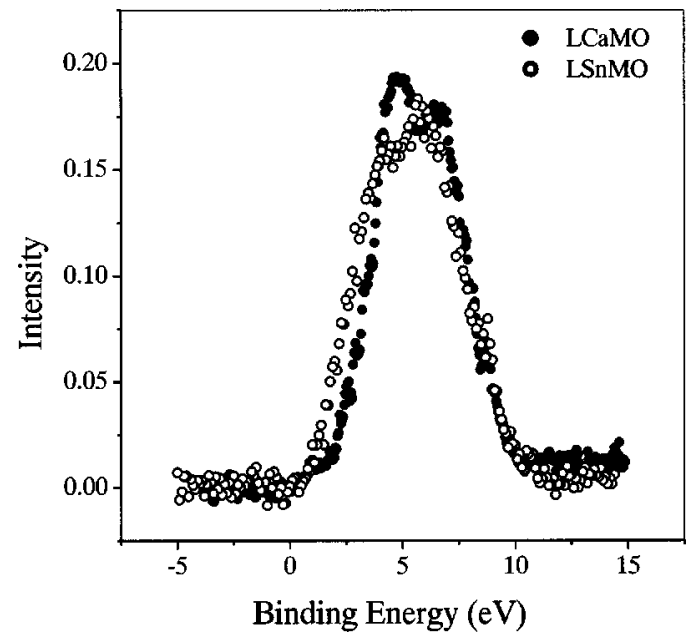

FIG. 4. Valence-band spectra of the $\mathrm{La}_{0.9} \mathrm{Sn}_{0.1} \mathrm{MnO}_{3}$ and $\mathrm{La}_{0.9} \mathrm{Sn}_{0.1} \mathrm{MnO}_{3}$ films.

films thus confirmed that the anomalous Hall coefficients are positive and the ordinary Hall coefficients are negative, different from the behavior of the divalent elements doping manganese perovskite oxides. ${ }^{8}$ The negative linear slope of the curves differs from those hole-doped $A B \mathrm{O}_{3}$ compounds. The carrier density turns out to be temperature dependent rather than field dependent. At $60 \mathrm{~K}$ we assume that the field dependence of Hall resistivity is a linear function; the Hall coefficient is about $-4.77 \mathrm{~cm}^{3} / \mathrm{C}$, and the carrier density is about $-0.13 \times 10^{19} \mathrm{~cm}^{3}$. For the temperature of $236 \mathrm{~K}$, that is near the Curie temperature, the magnetic-field dependence of the Hall resistivity exhibited activated behavior, which was observed in $\mathrm{La}_{2 / 3} \mathrm{Ca}_{1 / 3} \mathrm{MnO}_{3}$ in the region of high temperature. ${ }^{10}$

As discussed above, LSMO shows different behaviors when compared with the hole-doping LCMO. Further evidence may be derived from the XPS measurements. Al- $K \alpha$ radiation was used and the scanning step was $0.05 \mathrm{eV}$. The background yielded by the second electrons was subtracted for clarity and the intensity was normalized to integrated intensity. All spectra were fitted with a Gaussian function. Figure 4 presents the valence-band spectra of LSMO and LCMO. The fine structure of the peaks is similar to other manganites, which is due to the nonbonding and bonding states of the hybridization between $\mathrm{Mn}-3 d$ and $\mathrm{O}-2 p$ electrons. ${ }^{11}$ A variance of bandwidth and a chemical shift of the valence-band top, $\sim 0.4 \mathrm{eV}$ towards to the Fermi level, are observed. Compared to LCMO, the bandwidth was widened for LSMO. These phenomena are consistent with the relative variation of the valence band of LSMO ( $n$ type) and LCMO ( $p$ type), though both compounds were in the mixedvalence states of $\mathrm{Mn}^{+2}$ and $\mathrm{Mn}^{+3} \cdot{ }^{13}$ In previous works, it has been reported that the Fermi level and bandwidth were functions of temperature, doping level, and pressure. ${ }^{14}$ For hole-doped manganese oxides, as the average radius of rareearth-site ions decreases, the Mn-O-Mn bond angle decreases, which reduces the bandwidth. ${ }^{13}$ The ionic radius of $\mathrm{Sn}^{4+}$ is smaller than that of $\mathrm{Ca}^{2+}$, while the bandwidth of LSMO is wider than that of LCMO, which suggests that the effects of Sn doping might be a factor dominating the band
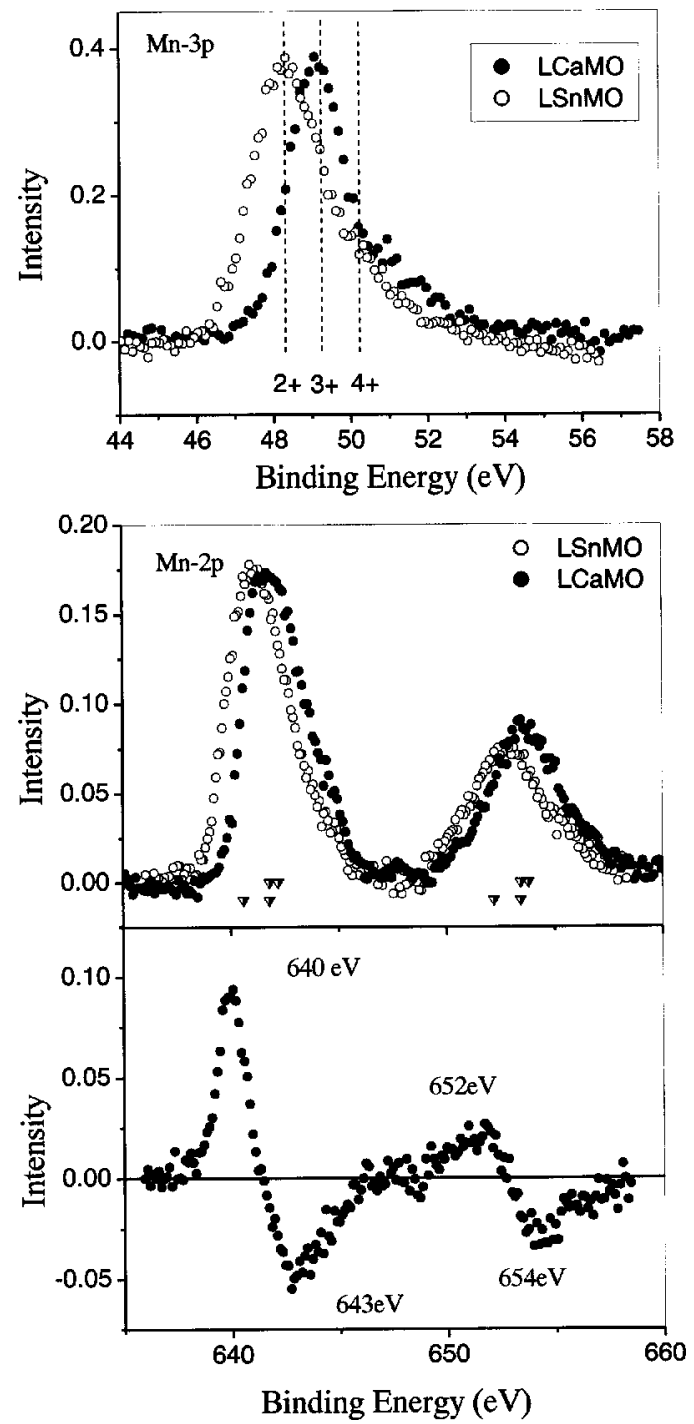

FIG. 5. XPS spectra of Mn-3p and Mn- $2 p$ core level, and the difference between the Mn-2p spectra of $\mathrm{La}_{0.9} \mathrm{Sn}_{0.1} \mathrm{MnO}_{3}$ and $\mathrm{La}_{0.9} \mathrm{Ca}_{0.1} \mathrm{MnO}_{3}$.

structure. Figure 5 shows the XPS pattern of Mn $3 p$ and $2 p$ and the difference of the Mn- $2 p$ spectra of LSMO and LCMO. The binding energy of $\mathrm{Mn}^{2+}, \mathrm{Mn}^{3+}$, and $\mathrm{Mn}^{4+}$ ions is marked by dashed lines for clarity. ${ }^{15}$ The XPS patterns of Mn- $2 p$ and $-3 p$ showed that the bandwidth of the LSMO sample is slightly wider than that of the hole-doped one, and that the spectrum of the LSMO film shifted in relatation to LCMO to a lower binding energy. Similar variances of the La- $3 d$, La- $4 d$, and O- $1 s$ spectra were also observed. Oku and Hirokawa pointed out that the difference between the binding energy of the $\mathrm{Mn}^{3+}$ - and $\mathrm{Mn}^{4+}-2 p$ level is only about $0.4 \mathrm{eV}$, while there is $1.3 \mathrm{eV}$ between that of $\mathrm{Mn}^{2+}$ and $\mathrm{Mn}^{3+}$ in manganese oxides. ${ }^{16}$ As a result, the bandwidth of the $\mathrm{Mn}^{2+} / \mathrm{Mn}^{3+}$ mixed-valence system could be wider than that of the $\mathrm{Mn}^{3+} / \mathrm{Mn}^{4+}$ system. Now the Mn- $2 p_{3 / 2}$ bandwidth (the line width at half maximum) of LSMO and LCMO is about 2.79 and $2.65 \mathrm{eV}$, respectively. The Mn-3p spectrum is asymmetric. A double fit found that the bandwidths are about 1.66 and $1.23 \mathrm{eV}$ for both compounds. This 
also implies that the two compounds are in different mixedvalence states. In addition, the peak positions of Mn- $2 p_{3 / 2}$ and $-3 p$ in the LSMO film are found about 641.35 and 48.22 $\mathrm{eV}$, respectively. The corresponding values for LCMO are about 642.13 and $49.05 \mathrm{eV}$. A chemical shift of about -0.8 $\mathrm{eV}$ in the Mn- $3 p$ and $-2 p$ levels could be found. In order to explore the variance between Mn- $2 p$ spectra, the difference between Mn- $2 p$ spectra of the LSMO and LCMO films was also plotted in Fig. 5. There are two positive peaks at about 640 and $652 \mathrm{eV}$, and two negative peaks at about 643 and $654 \mathrm{eV}$. The former two peaks are very close to that of $2 p_{3 / 2}$ and $2 p_{1 / 2}$ states of $\mathrm{MnO}(640.6$ and $652.2 \mathrm{eV})$, while the latter two to the peaks of the $2 p_{3 / 2}$ and $2 p_{1 / 2}$ states of $\mathrm{MnO}_{2}$ $(642.2$ and $653.8 \mathrm{eV}){ }^{12}$ This implies that the number of $\mathrm{Mn}^{4+}$ ions decreased or vanished and the $\mathrm{Mn}^{2+}$ appeared in LSMO. Therefore, the $\mathrm{Mn}^{2+}$ ion may replace $\mathrm{Mn}^{4+}$ due to the substitution of $\mathrm{Sn}$ for $\mathrm{Ca}$ in the compound. That is to say,
Mn ions in LSMO are at a $\mathrm{Mn}^{2+} / \mathrm{Mn}^{3+}$ mixed-valence state, different from the conventional CMR materials that show a $\mathrm{Mn}^{3+} / \mathrm{Mn}^{4+}$ mixed-valence state.

In conclusion, the experimental results of this XPS study revealed that the $\mathrm{Sn}$ ions were in the four-valence state in the LSMO films. In comparison to LCMO films, the bandwidth of valence band was widened and the Fermi energy level was shifted by about $-0.4 \mathrm{eV}$. The difference curve between the Mn- $2 p$ spectrum of LSMO and that of LCMO showed that the substitution of $\mathrm{Sn}$ for $\mathrm{Ca}$ may transfer manganese perovskite from the $\mathrm{Mn}^{3+} / \mathrm{Mn}^{4+}$ mixed-valence system into an $\mathrm{Mn}^{2+} / \mathrm{Mn}^{3+}$ one, which implies that the mechanism for the CMR behavior could be different from that the hole-doped manganites.

We thank Professor J. R. Sun for helpful discussions. This work was supported by a grant from the Research Grant Council of Hong Kong, China.
${ }^{1}$ A. P. Ramirez, J. Phys.: Condens. Matter 9, 8171 (1997); C. N. R. Rao, Philos. Trans. R. Soc. London, Ser. A 356, 23 (1998); J. A. Alonso, ibid. 356, 1617 (1998); J. M. D. Coey, M. Vret, and S. Von Molnar, Adv. Phys. 48, 167 (1999).

${ }^{2}$ C. Zener, Phys. Rev. 82, 403 (1951); P. W. Anderson and H. Hasegawa, ibid. 100, 675 (1955); J. B. A. A. Elemans, B. Van Laar, K. R. Van Der Veen, and B. O. Loopstra, J. Solid State Chem. 3, 238 (1971); A. J. Millis, B. I. Shaiman, and R. Mueller, Phys. Rev. Lett. 77, 175 (1996).

${ }^{3}$ A. Maignan, C. Martin, and B. Raveau, Chem. Mater. 10, 950 (1998); Z. Phys. B: Condens. Matter 102, 19 (1997).

${ }^{4}$ Z. W. Lin, A. H. Morrish, X. Z. Xhou, and S. Dai, J. Appl. Phys. 83, 7398 (1998); A. Caneiro, L. Morales, F. Prado, D. G. Lamas, R. D. Sanchez, and A. Serquis, Phys. Rev. B 62, 6825 (2000).

${ }^{5}$ Xiangxin Guo, S. Dai, Y. Zhou, G. Yang, and Z. Chen, Appl. Phys. Lett. 75, 3378 (1999); X. Guo, Z. Chen, S. Dai, Y. Zhou, R. Li, H. Zhang, B. Shen, and H. Cheng, J. Appl. Phys. 88, 4768 (2000).

${ }^{6}$ A. Gupta, T. R. McGuire, P. R. Duncombe, M. Rupp, J. Z. Sun, W. J. Gallagher, and Gang Xiao, Appl. Phys. Lett. 67, 3494 (1995).

${ }^{7}$ T. R. McGuire, A. Gupta, P. F. Duncombe, M. Rupp, J. Z. Sun, R. B. Laibowitz, W. J. Gallagher, and Gang Xao, J. Appl. Phys. 79, 4549 (1996).

${ }^{8}$ A. W. C. Lin, N. R. Armsrong, and T. Kuwana, Anal. Chem. 49,
1228 (1977); H. Willemen, D. F. Van De Vondel, and G. P. Van De Kclen, Inorg. Chim. Acta 34, 175 (1979).

${ }^{9}$ I. Imai, Y. Shimakawa, Yu. Sushko, and Y. Kubo, Phys. Rev. B 62, 12190 (2000); Yeongkuo Lin, Dean J. Miller, J. S. Jiang, John Pearson, and S. D. Bader, J. Appl. Phys. 87, 5576 (2000).

${ }^{10}$ Y. Lyanda-Geller, S. H. Chun, M. B. Salamon, P. M. Goldbart, P. D. Han, Y. Tomioka, A. Asaitsu, and Y. Tokura, Phys. Rev. B 63, 184426 (2001).

${ }^{11}$ I. Gordon, P. Wagner, A. das, J. Vanacken, V. V. Moschalkov, Y. Bruynseraede, W. Schuddinck, G. Van Tendeloo, M. Ziese, and G. Borghs, Phys. Rev. B 62, 11633 (2000).

${ }^{12}$ T. Saitoh, A. Sekiyama, K. Kobayashi, T. Mizokawa, A. Fujimoti, D. D. Sanna, Y. Takeda, and M. Takano, Phys. Rev. B 56, 8836 (1997).

${ }^{13}$ T. Saitoh, A. E. Bocquet, T. Mizokawa, H. Namatame, A. Fujimori, M. Abbate, Y. Takeda, and M. Takano, Phys. Rev. B 51, 13942 (1995).

${ }^{14}$ J. H. Park, C. T. Chen, S-W. Cheong, W. Bao, G. Meigs, V. Chakarian, and Y. U. Idzerda, Phys. Rev. Lett. 22, 4315 (1996); Y. Moritomo, A. Asamitsu, and Y. Tokura, Phys. Rev. B 56, 12190 (1997).

${ }^{15}$ J. B. Torrance, P. Lacorre, A. I. Nazzal, E. J. Ansaldo, and Ch. Niedermayer, Phys. Rev. B 45, 8209 (1992); Y. Tokura, J. Appl. Phys. 79, 5288 (1996).

${ }^{16}$ Masaoki Oku, Kichinosuke Hirokawa, and Shigero Ikeda, J. Electron Spectrosc. Relat. Phenom. 7, 465 (1975). 